\title{
Ultraviolet to near-infrared spectroscopy of the potentially hazardous, low delta-V asteroid (175706) 1996 FG3
}

\section{Backup target of the sample return mission MarcoPolo- $R^{\star}$}

\author{
D. Perna ${ }^{1}$, E. Dotto ${ }^{2}$, M. A. Barucci ${ }^{1}$, S. Fornasier ${ }^{1,3}$, A. Alvarez-Candal ${ }^{4,5,6}$, F. Gourgeot ${ }^{1}$, \\ J. R. Brucato ${ }^{7}$, and A. Rossi ${ }^{8}$
}

\author{
${ }^{1}$ LESIA - Observatoire de Paris, CNRS, UPMC Univ. Paris 06, Univ. Paris-Diderot, 5 place J. Janssen, 92195 Meudon, France \\ e-mail: davide.perna@obspm.fr \\ 2 INAF - Osservatorio Astronomico di Roma, via Frascati 33, 00040 Monte Porzio Catone, Roma, Italy \\ 3 Univ. Paris Diderot, Sorbonne Paris Cité, 4 rue Elsa Morante, 75205 Paris, France \\ ${ }^{4}$ Observatório Nacional, COAA, Rua Gal. José Cristino 77, 20921-400 Rio de Janeiro, Brazil \\ 5 Instituto de Astrofísica de Andalucía - CSIC, Glorieta de la Astronomía s/n, 18008 Granada, Spain \\ ${ }^{6}$ European Southern Observatory, Alonso de Córdova 3107, Vitacura, Casilla 19001, Santiago 19, Chile \\ 7 INAF - Osservatorio Astrofisico di Arcetri, L.go E. Fermi 5, 50125 Firenze, Italy \\ 8 IFAC - CNR, Via Madonna del Piano 10, 50019 Sesto Fiorentino (FI), Italy
}

Received 10 April 2013 / Accepted 16 May 2013

\section{ABSTRACT}

\begin{abstract}
Context. Primitive near-Earth asteroids (NEAs) are important subjects of study for current planetary research. Their investigation can provide crucial information on topics such as the formation of the solar system, the emergence of life, and the mitigation of the risk of asteroid impact. Sample return missions from primitive asteroids have been scheduled or are being studied by space agencies, including the MarcoPolo- $R$ mission selected for the assessment study phase of ESA M3 missions.

Aims. We want to improve our knowledge of the surface composition and physical nature of the potentially hazardous, low delta-V asteroid (175706) 1996 FG3, backup target of MarcoPolo- $R$. This intriguing object shows an as-yet unexplained spectral variability. Methods. We performed spectroscopic observations of 1996 FG3 using the visible spectrograph DOLORES at the Telescopio Nazionale Galileo (TNG), and the UV-to-NIR X-Shooter instrument at the ESO Very Large Telescope (VLT).

Results. We find featureless spectra and we classify 1996 FG3 as a primitive Xc-type in the Bus-DeMeo taxonomy. Based on literature comparison, we confirm the spectral variability of this object at near-infrared (NIR) wavelengths, and find that spectral variations exist also for the visible spectral region. Phase reddening cannot explain such variations. Obtained with the same observational conditions for the whole 0.3-2.2 $\mu \mathrm{m}$ range, our X-Shooter spectrum allowed a proper comparison with the RELAB meteorite database. A very good fit is obtained with the very primitive C2 Tagish Lake carbonaceous chondrite (pressed powder), confirming 1996 FG3 as a suitable target for a sample return mission from primitive NEAs.

Conclusions. We hypothesize a compacted/cemented surface for 1996 FG3, like that observed by the Hayabusa mission on (25143) Itokawa, with the possible presence of regions showing different degrees of surface roughness. This variegation could be related to the binary nature of 1996 FG3, but to check this hypothesis further observations are necessary.
\end{abstract}

Key words. minor planets, asteroids: individual: (175706) 1996 FG3 - techniques: spectroscopic

\section{Introduction}

For a number of reasons, the investigation of primitive, lowalbedo near-Earth asteroids (NEAs) represents one of the main topics of current planetary research. The primitive NEA population is continuously replenished via powerful and diffusive resonances (e.g., Morbidelli et al. 2002) from the outer main belt, where more pristine water- and organic-rich material lies than in the more thermally evolved silicaceous asteroid population which dominates the inner main belt. However, we note

* Based on observations carried out at the European Southern Observatory (ESO), Chile (programme 088.C-0695), and with the Italian Telescopio Nazionale Galileo (TNG) operated on the island of La Palma by the Fundacion Galileo Galilei of the INAF (Istituto Nazionale di Astrofisica) at the Spanish Observatorio del Roque de los Muchachos of the Instituto de Astrofisica de Canarias (programme AOT25/TAC13). that some primitive NEAs (including 1996 FG3) are compatible with an origin from the so-called Nysa-Polana complex in the inner main belt (Walsh et al. 2013). Some low-albedo NEAs, like 1999 JU3, 1999 RQ36, and 2008 EV5, have been associated (Moskovitz et al. 2013; Clark et al. 2011; Reddy et al. 2012) with the primitive carbonaceous chondrite meteorites (which contain the first solids to condense in the solar system), and these objects probably had a major role in the origin of life on our planet, with the delivery of water and complex organic molecules to the early Earth that could have triggered the pre-biotic synthesis of biochemical compounds (e.g., Morbidelli et al. 2000). Primitive potentially hazardous asteroids (PHAs) could also pose a special hazard to our planet and the most promising mitigation techniques, such as the kinetic impactor or the nuclear blast deflection (see, e.g., Harris et al. 2012), would have to deal with the strong dissipative effect intrinsic of such low-density porous bodies evidenced by laboratory experiments 
of collisional fragmentation, numerical simulations, and scaling theories (e.g., Housen \& Holsapple 2012).

For all of the above reasons, sample returns from primitive NEAs have emerged as a fundamental goal to be accomplished, and all of the major space agencies are planning such missions: the NASA OSIRIS-REx (launch in 2016, return to Earth in 2023) will sample the asteroid (101955) 1999 RQ36, while the JAXA Hayabusa2 mission (launch in 2014) will bring back material from the NEA (162173) 1999 JU3 in 2020. At a European level, the European Space Agency (ESA) inserted direct laboratory analysis of samples returned by a NEA among the major topics to be investigated in the Cosmic Vision 2015-2025 timeframe. In this context, the MarcoPolo- $R$ (MP-R) space mission, aimed at visiting a primitive NEA, scientifically characterize it, and bring samples back to the Earth, has been selected for an assessment study with a launch between 2022 and 2024 (Barucci et al. 2012).

The PHA (175706) 1996 FG3 is currently the backup target of MP-R; it was previously the main target of the mission until December 2012. Even if it will be not visited by MP-R, this object represents a very appealing target for a space mission. In the past it was already the primary target of the Leonard mission study at the French space agency CNES, and of the Galahad mission proposed for the NASA New Frontier program. Its orbital properties (semi-major axis of $1.054 \mathrm{AU}$, eccentricity of 0.35 , inclination of $2^{\circ}$ ) make it an easily accessible target for a spacecraft, with a velocity increment required for a spacecraft starting from the Earth's orbit to reach the asteroid of only $\Delta V=3.07 \mathrm{~km} \mathrm{~s}^{-1}$ (Christou 2003). The physical properties of 1996 FG3 also seem extremely intriguing. It is a binary object, which allows precise estimates for the sizes, mass, and orbit of the system on the basis of ground-based observations (with the advantage of a better pre-launch optimization of the mission design and operations). Both lightcurve studies (Pravec et al. 2006; Scheirich \& Pravec 2009) and radar observations (Benner et al. 2012) indicate a primary of about $1.7-1.8 \mathrm{~km}$ and a secondary elongated and roughly $500 \mathrm{~m}$ in diameter, orbiting with a semimajor axis $\gtrsim 2.5 \mathrm{~km}$ (Benner et al. 2012). The rotational period of the primary is $3.6 \mathrm{~h}$ (Pravec et al. 2006), while Scheirich \& Pravec (2009) found that the secondary body has a synchronous (or nearly so) rotation with an orbital period of $16.1 \mathrm{~h}$ and also found a pole solution $\lambda_{\mathrm{p}}=242^{\circ} \pm 96^{\circ}, \beta_{\mathrm{p}}=-84_{-5^{\circ}}^{\circ}$, and a bulk density of $1.4_{-0.6}^{+1.5} \mathrm{~g} \mathrm{~cm}^{-3}$. Preliminary results from radar observation suggest a low value for the density, roughly around $0.9 \mathrm{~g} \mathrm{~cm}^{-3}$ (Benner et al. 2012). The binary nature of this body can also give crucial information that may allow discrimination between the most likely formation mechanisms, as well as a unique opportunity to study the dynamical evolution driven by the YORP/Yarkovsky thermal effects. It has been shown that tidal disruption should account for approximately $1-2 \%$ of NEAs being binaries, while the estimated binary fraction among NEAs is $\sim 15 \%$ (Walsh \& Richardson 2008). Walsh et al. (2008) showed that the most likely formation mechanism for NEA binaries is the slow spin-up of a rubble pile asteroid by means of the thermal YORP effect, and the shape and rotation period of the primary, orbital and rotational period of the secondary, as well as the orbital semi-major axis and eccentricity, can be used as model parameters (Walsh et al. 2012b). Multiwavelength photometric observations allowed Wolters et al. (2011) to determine an absolute magnitude $H_{v}=17.833 \pm 0.024$, a phase parameter $G=-0.041 \pm 0.005$, an albedo $p_{v}=0.044 \pm 0.004$, a primary mean spherical diameter $D_{\mathrm{p}}=1.69_{-0.12}^{+0.18} \mathrm{~km}$, a secondary diameter $D_{\mathrm{s}}=0.51 \pm 0.03 \mathrm{~km}$, a secondary orbital semi-major axis $a=2.8_{-0.7}^{+1.7} \mathrm{~km}$, and a thermal inertia $\Gamma=120 \pm 50 \mathrm{~J} \mathrm{~m}^{-2} \mathrm{~s}^{-1 / 2} \mathrm{~K}^{-1}$ (suggesting a dusty surface), and to predict a Yarkovsky drift in semi-major axis of $-60_{-45}^{+31} \mathrm{~m} \mathrm{yr}^{-1}$.

The spectral properties of 1996 FG3 are still under debate. Its only published visible spectrum was obtained in 2001 in the framework of the SMASS survey and this NEA was classified as a primitive B- or C-type object (Binzel et al. 2001). Nearinfrared spectra obtained by several authors (de León et al. 2011; Binzel et al. 2012; Walsh et al. 2012a) are still consistent within the C-complex, but exhibit significant differences in the spectral slope. Observations in the $3 \mu \mathrm{m}$ spectral region (Rivkin et al. 2013) showed an absorption band of $\sim 5-10 \%$, a diagnostic for hydrated/hydroxylated minerals on the surface. This indicates that the thermal heating was not intense enough to reach dehydrating temperatures, and that relatively fresh areas exist on the surface of 1996 FG3. The asteroid 1996 FG3 is only the second NEA on which a $3 \mu \mathrm{m}$ feature has been observed, after 1992 UY4 (Volquardsen et al. 2007).

To improve our knowledge of the surface composition and nature of this object, we performed spectroscopic observations at the Italian Telescopio Nazionale Galileo (TNG) of the European Northern Observatory (ENO) in La Palma, Spain, and at the Very Large Telescope (VLT) of the European Southern Observatory (ESO), Chile.

\section{Observations and data reduction}

Visible observations were performed in February 2012 at the ENO-TNG. We used the Device Optimized for the LOw RESolution (DOLORES) instrument equipped with the lowresolution red grism (LR-R) covering the $\sim 0.50-0.95 \mu \mathrm{m}$ range, with a spectral dispersion of $2.6 \AA / \mathrm{px}$, and using a slit width of $2^{\prime \prime}{ }^{1}$. The obtained visible spectrum was reduced with the software package Midas, using ordinary procedures of data reduction (e.g., Fornasier et al. 2008; Perna et al. 2010) which include the subtraction of the bias from the raw data, flat-field correction, cosmic ray removal, sky subtraction, collapsing the twodimensional spectra to one dimension, wavelength calibration, and atmospheric extinction correction. The reflectivity was obtained by dividing the spectrum by that of a solar analog star observed closest in time and airmass to the object.

A wider spectral range from $0.3 \mu \mathrm{m}$ to $2.2 \mu \mathrm{m}$ was obtained at the ESO-VLT using the Echelle spectrograph X-Shooter, described at the ESO webpage ${ }^{2}$. This instrument allowed us to cover simultaneously the UV+optical+NIR wavelength range without introducing the usual uncertainties due to the combination of different band spectra (by means of multiband photometric observations used to calibrate and align the different spectroscopic ranges) acquired at different epochs, and therefore at different observational conditions and rotational phases. Observations were carried out in January 2012. We chose the SLIT mode, selecting the high-gain readout mode and $2 \times 1$ binning for the UVB and VIS detectors. The read-out and binning for the near-infrared (NIR) detector are fixed. The slit widths were $1.0^{\prime \prime}, 0.9^{\prime \prime}$, and $0.9^{\prime \prime}$, for the UVB, VIS, and NIR arms, respectively, yielding a resolving power of about 5000 per arm. As usual with NIR observations, we nodded on the slit to remove the sky contribution. To remove the solar and telluric signals from 1996 FG3 spectrum, we observed a solar analog with the same observational setup as our target and at similar airmass to

\footnotetext{
http://www.tng.iac.es/instruments/lrs/

2 http://www . eso.org/sci/facilities/paranal/ instruments/xshooter/
} 
Table 1. Observational circumstances.

\begin{tabular}{lccccc}
\hline \hline Telescope & Date & $\begin{array}{c}\mathrm{UT}_{\text {start }} \\
(\mathrm{hh}: \mathrm{mm})\end{array}$ & $\begin{array}{c}T_{\exp } \\
(\mathrm{s})\end{array}$ & Airmass & Solar analog (airm.) \\
\hline TNG & 2012 Feb. 18 & $22: 34$ & $4 \times 600$ & $1.08-1.23$ & Land98-978 (1.25) \\
VLT & 2012 Jan. 13 & $01: 46$ & $8 \times 280$ & $1.56-1.55$ & Hyades64 (1.33) \\
\hline
\end{tabular}

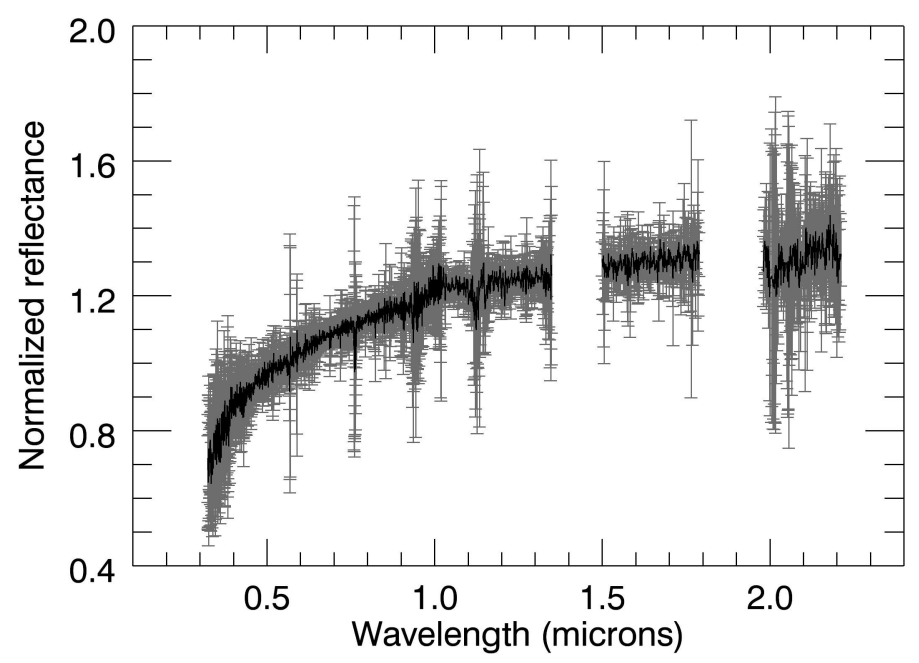

Fig. 1. Obtained VLT spectrum (gray). The smoothed data are shown in black. Spectral regions affected by strong atmospheric absorption or low instrumental efficiency have been cut out.

minimize the effects of differential refraction. The data were reduced using the X-Shooter pipeline. We followed the procedure described in Alvarez-Candal et al. (2011), which includes flatfielding, wavelength calibration, merging of different orders, and the extraction of the spectrum. The data were wavelength and spatially calibrated by creating a two-dimensional wave-map, necessary because of the curvature of the Echelle orders. Then we extracted the one-dimensional spectra and we divided those of 1996 FG3 by the corresponding star, used as a telluric and solar analog star. Finally, a median filter with a window of 20 pixels and threshold of 0.08 in reflectance was applied to smooth the spectrum.

Details of both TNG and VLT observations are given in Table 1 . The obtained spectra, normalized to unity at $0.55 \mu \mathrm{m}$, are presented in Figs. 1 and 3.

\section{Spectral analysis and discussion}

We obtained spectra with no absorption features. All of the features that can be seen in our spectra, e.g., at $\sim 1.12 \mu \mathrm{m}$ and at $\sim 2 \mu \mathrm{m}$, are artifacts introduced by the star and/or poor telluric correction. The drop in the reflectance that can be seen for wavelengths below $\sim 0.4 \mu \mathrm{m}$ is a common feature among C-type asteroids, and it is usually attributed to $\mathrm{Fe}^{2+} \rightarrow \mathrm{Fe}^{3+}$ transitions in iron-bearing hydrated silicates (see, e.g., Rivkin et al. 2002).

As stated in Sect. 1, 1996 FG3 shows a considerable variability in terms of NIR spectral slope. Our X-Shooter spectrum continues in this trend, with an intermediate NIR slope (Fig. 2) between the redder and bluer spectra of Binzel et al. (2012), which represent the two color extremes in the literature. We can now extend the already known NIR spectral variability to visible wavelengths (Fig. 3), as both our TNG and VLT data present a much redder slope in this region than the only visible spectrum available in the literature so far (Binzel et al. 2001). Though very similar up to $\sim 0.55 \mu \mathrm{m}$, the X-Shooter spectrum

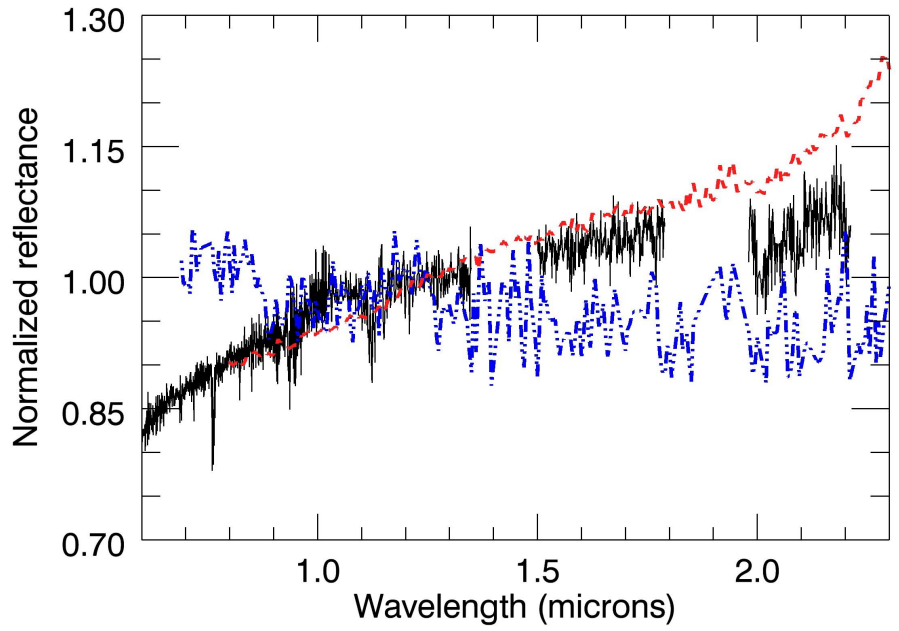

Fig. 2. NIR variability. The VLT smoothed spectrum is shown in black. Red (dashed) and blue (dot-dot-dashed) spectra are from Binzel et al. (2012), and were acquired on 2011 Dec. 1 and 2009 Apr. 27, respectively. All the spectra are normalized at $1.25 \mu \mathrm{m}$.

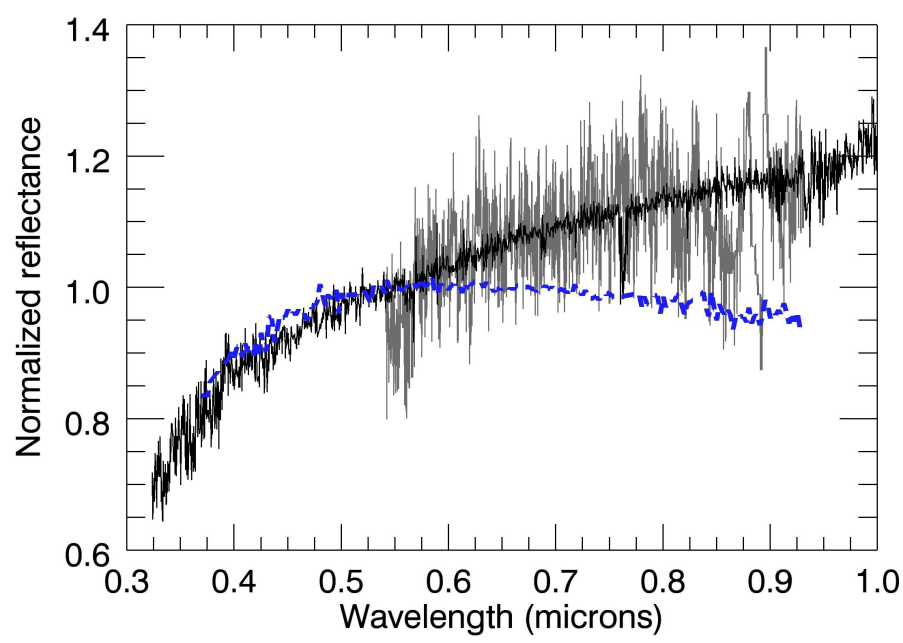

Fig. 3. Visible variability. The VLT smoothed spectrum is shown in black, our TNG spectrum in gray, the visible spectrum from Binzel et al. (2001) with a blue dashed line.

has a spectral slope (computed between $0.6 \mu \mathrm{m}$ and $0.9 \mu \mathrm{m}$ ) of $(4.3 \pm 0.1) \% / 10^{3} \AA$, against a slope of $(-1.7 \pm 0.1) \% / 10^{3} \AA$ for the visible spectrum by Binzel et al. (2001). The TNG and VLT data were obtained at quite different phase angles $\left(41.6^{\circ}\right.$ and $26.7^{\circ}$, respectively), but present the same spectral slope in the visible, which means that the spectral variation with respect to the data from Binzel et al. (2001), obtained at a phase angle of $2.8^{\circ}$, probably cannot be explained by phase reddening. We stress, however, that phase reddening is not yet well understood (see Dahlgren et al. 1997; Michelsen et al. 2006; Sanchez et al. 2012, and references therein), especially for primitive asteroid types. Lumme \& Bowell (1981), for example, report a mean phase reddening for C-type asteroids of $(0.15 \pm 0.12) \% / 10^{3} \AA /$ degree in $B-V$, while Dahlgren et al. (1997) find that the phase reddening effect is negligible for the taxonomic $\mathrm{P}$ and $\mathrm{D}$ classes. The spectral variability at visible wavelengths seems likely to be correlated with the already known variations in the NIR spectral range; unfortunately, no explanation has been found for this. A number of possible physical causes have been already checked and ruled out (DeMeo, pers. comm.): cometary activity, satellite 
of a different color, phase reddening, post-perihelion thermal effect. Additional explanations to account for the observed variability could rely on a variegated surface. A careful investigation of possible relationships between the spectral slope and the rotational phase and/or the aspect angle (all of the available data of 1996 FG3 were acquired with a nearly equatorial view) is still missing, and additional dedicated observations are required. For instance, at the beginning of 2014 the asteroid will have a magnitude $V \sim 20$ and will be observable during the first hours of the night from Chile, Hawaii, and the Canary Islands, for example, where different telescopes of the 8-10 m class lie. This would be the occasion to take good quality rotationally resolved spectra using exposure times which cover only a small fraction of the primary spin period.

For the taxonomic classification and the meteorite comparison of our data, we used a code based on chi-square minimization. We checked our findings against the M4AST online tool ${ }^{3}$ (Popescu et al. 2012) and obtained the same results. In agreement with the results obtained for previous spectra of 1996 FG3 (see de León et al. 2011), we find that several taxa belonging to the primitive $\mathrm{X}$ - and C-complex (e.g., Xc, Xk, Cgh) defined by the Bus-DeMeo taxonomy (DeMeo et al. 2009) are considered to be plausible classifications by the automatic procedure, with similar confidence level. Nonetheless, the absence of distinctive absorption features in our spectra around $0.7 \mu \mathrm{m}$ and $1 \mu \mathrm{m}$ points towards a classification of Xc-type.

We stress the importance of our new X-Shooter spectrum in the search for good spectral analogs for 1996 FG3 within the existing meteorite collection: a truly reliable comparison with laboratory spectra requires that the surface of the asteroid be investigated with the same observational conditions, including rotational phase, aspect angle, phase angle, airmass, etc., for the whole spectral range. With this strong advantage, we compared our X-Shooter spectrum with the RELAB spectral database (Pieters \& Hiroi 2004), using the November 2011 release. Taking into consideration the information on the very low albedo of 1996 FG3, we focused our search on carbonaceous chondrites as likely analogs of the asteroid, for a total of about 600 spectra found in the RELAB database. The only meteorite for which we obtain a good fit for the whole $0.3-2.2 \mu \mathrm{m}$ range is the Tagish Lake (Fig. 4), in the form of pressed powder (grain size $<125 \mu \mathrm{m})$. This association is strengthened by the recent detection of the $3 \mu \mathrm{m}$ feature in the spectrum of 1996 FG3 (Rivkin et al. 2013). Indeed, though deeper and different in shape, a $3 \mu \mathrm{m}$ absorption band is present also in the spectrum of the Tagish Lake meteorite (Hiroi et al. 2001). The Tagish Lake C2 (ungrouped) carbonaceous chondrite is considered one of the most primitive solar system materials yet studied. Its mineralogical and chemical properties are between those of CM and CI meteorites, and its high microporosity $(\sim 40 \%)$ makes it a good candidate material for the composition of low bulk density primitive asteroids (e.g., Brown et al. 2000; Hildebrand et al. 2006). Moreover, nonterrestrial L-proteinogenic amino acid excesses have been detected in this meteorite, strengthening the hypothesis that primitive minor bodies could have delivered enantiomerically enriched amino acids to the early Earth that could have triggered the emergence of life (Glavin et al. 2012). If confirmed, a link with the Tagish Lake meteorite would be fascinating, and would confirm the very primitive nature of 1996 FG3, in agreement with the results by Rivkin et al. (2013).

The spectrum of a fresh, not pressed, sample of the Tagish Lake meteorite shows a redder behaviour (Fig. 4).

3 http://cardamine.imcce.fr/m4ast/

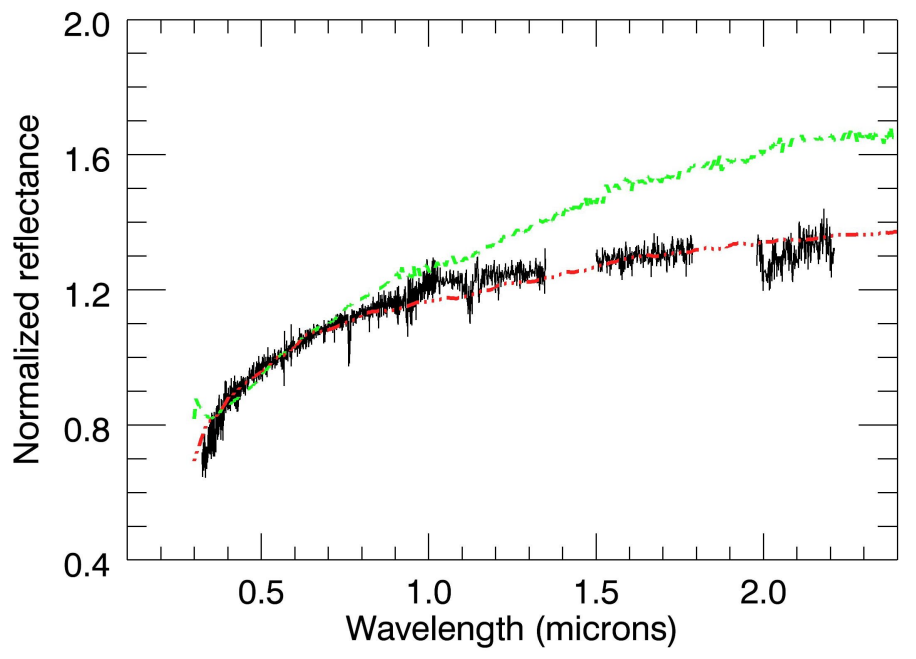

Fig. 4. Meteorite comparison. In black, our X-Shooter smoothed spectrum. In red (dot-dot-dashed), the spectrum of the Tagish Lake meteorite, as pressed powder (RELAB sample MT-TXH-025-L0). In green (dashed), the spectrum of a relatively fresh portion of the Tagish Lake meteorite (RELAB sample MT-MEZ-011). For both meteorite samples, grain size is $<125 \mu \mathrm{m}$.

This might lead us to speculate that we were looking at a compacted/cemented surface on 1996 FG3, like that observed on (25143) Itokawa by the Hayabusa mission (Saito et al. 2006). This seems to agree with the results from radar observations by Benner et al. (2012), who found for 1996 FG3 a circular polarization ratio $\mathrm{SC} / \mathrm{OC}=0.34 \pm 0.02$, similar to that of Itokawa $(0.28 \pm 0.04)$, suggesting a comparable degree of near-surface roughness. The evidence for dust coming from the quite low thermal inertia could support the hypothesis of the presence of both rough and smooth terrains even on 1996 FG3.

\section{Conclusions}

We present new spectra of the potentially hazardous, low delta-V asteroid (175706) 1996 FG3, backup target of the sample return mission MarcoPolo- $R$, currently in competition for selection by ESA in the framework of the Cosmic Vision 2015-2025 timeframe. A visible spectrum has been acquired at the TNG telescope, while UV-to-NIR $(0.3-2.2 \mu \mathrm{m})$ spectroscopy has been obtained with the X-Shooter instrument at the VLT. Our data suggest a classification of 1996 FG3 as a featureless, primitive Xc-type according to the Bus-DeMeo taxonomy. We confirm that 1996 FG3 presents a considerable variation of its spectral properties in the NIR; this variability extends to visible wavelengths, and we exclude that phase reddening could explain it. Our X-Shooter spectrum, with UV-to-NIR information obtained with the same observational conditions, allowed a proper comparison with laboratory meteorite spectra, for which we used the RELAB database. We find the best match with the anomalous Tagish Lake carbonaceous chondrite, in the form of pressed powder, confirming the very primitive nature of 1996 FG3. We hypothesize an Itokawa-like compacted/cemented surface for 1996 FG3, with the presence of regions showing different degrees of surface roughness. This variegation could be possibly related to the effect on the primary body of the 1996 FG3 system by its satellite, as recently suggested by Campo Bagatin et al. (2012), but further dedicated observations are needed to check this hypothesis.

The possible link with the Tagish Lake meteorite, as well as the intriguing science related to the spectral variability of 
1996 FG3, makes it a very suitable target for a space mission, and especially for a sample return, also in consideration of its accessible orbit and possible importance for the mitigation of the asteroid impact risk.

Acknowledgements. Part of this work was supported by INAF (PRIN-INAF 2009 "Near Earth Objects"), ASI (A.A. ASI I/079/9/0), and CNES funds. A.A.C. acknowledges support from the Marie Curie Actions of the European Commission (FP7-COFUND). Part of the data utilized in this publication were obtained and made available by The MIT-UH-IRTF Joint Campaign for NEO Reconnaissance. The IRTF is operated by the University of Hawaii under Cooperative Agreement No. NCC 5-538 with the National Aeronautics and Space Administration, Office of Space Science, Planetary Astronomy Program. The MIT component of this work is supported by NASA Grant 09NEOO009-0001, and previously by the National Science Foundation under Grant No. 0506716. Last but not least, we thank the anonymous referee whose comments helped us to improve the article.

\section{References}

Alvarez-Candal, A., Pinilla-Alonso, N., Licandro, J., et al. 2011, A\&A, 532, A130

Barucci, M. A., Cheng, A. F., Michel, P., et al. 2012, ExA, 33, 645

Benner, L. A. M., Brozovic, M., Giorgini, J. D., et al. 2012, 44th DPS meeting abstract, id. 102.06

Binzel, R. P., Harris, A. W., Bus, S. J., \& Burbine, T. H. 2001, Icarus 151, 139

Binzel, R. P., Polishook, D., DeMeo, F. E., Emery, J. P., \& Rivkin, A. S. 2012, 43rd LPSC abstract, 1659, 2222

Brown, P. G., Hildebrand, A. R., Zolensky, M. E., et al. 2000, Science, 290, 320

Campo Bagatin, A., Rossi, A., Alemañ, R. A., et al. 2012, EPSC 2012 abstract, id. 542

Christou, A. A. 2003, P\&SS, 51, 221

Clark, B. E., Binzel, R. P., Howell, E. S., et al. 2011, Icarus, 216, 462

Dahlgren, M., Lagerkvist, C.-I., Fitzsimmons, A., Williams, I. P., \& Gordon, M. 1997, A\&A, 323, 606

de León, J., Mothé-Diniz, T., Licandro, J., Pinilla-Alonso, N., \& Campins, H. 2011, A\&A, 530, L12
DeMeo, F. E., Binzel, R. P., Slivan, S. M., \& Bus, S. J. 2009, Icarus, 202, 160 Fornasier, S., Migliorini, A., Dotto, E., \& Barucci, M. A. 2008, Icarus, 196, 119 Glavin, D. P., Elsila, J. E., Burton, A. S., et al. 2012, M\&PS, 47, 1347

Harris, A. W., Barucci, M. A., Cano, J. L., et al. 2012, EPSC 2012 abstract, id. 126

Hildebrand, A. R., McCausland, P. J. A., Brown, P. G., et al. 2006, M\&PS, 41, 407

Hiroi, T., Zolensky, M. E., \& Pieters, C. M. 2001, Science, 293, 2234

Housen, K. R., \& Holsapple, K. A. 2012, Icarus, 219, 297

Lumme, K., \& Bowell, E. 1981, AJ, 86, 1705

Michelsen, R., Nathues, A., \& Lagerkvist, C.-I. 2006, A\&A, 451, 331

Morbidelli, A., Chambers, J., Lunine, J. I., et al. 2000, M\&PS, 35, 1309

Morbidelli, A., Bottke, W. F. J., Froeschlé, C., \& Michel, P. 2002, in Asteroids III, eds. W. F. J. Bottke, A. Cellino, P. Paolicchi, \& R. P. Binzel (Tucson: Univ. of Arizona Press), 409

Moskovitz, N. A., Abe, S., Pan, K.-S., et al. 2013, Icarus, 224, 24

Perna, D., Dotto, E., Lazzarin, M., et al. 2010, A\&A, 513, L4

Pieters, C. M., \& Hiroi, T. 2004, 35th LPSC abstract, id. 1720

Popescu, M., Birlan, M., \& Nedelcu, D. A. 2012, A\&A, 544, A130

Pravec, P., Scheirich, P., Kušnirák, P., et al. 2006, Icarus, 181, 63

Reddy, V., Corre, L. Le, Hicks, M., et al. 2012, Icarus, 221, 678

Rivkin, A. S., Howell, E. S., Vilas, F., \& Lebofsky, L. A. 2002, in Asteroids III, eds. W. F. J. Bottke, A. Cellino, P. Paolicchi, \& R. P. Binzel (Tucson: Univ. of Arizona Press), 235

Rivkin, A. S., Howell, E. S., Vervack, R. J., et al. 2013, Icarus, 223, 493

Saito, J., Miyamoto, H., Nakamura, R., et al. 2006, Science, 312, 1341

Sanchez, J. A., Reddy, V., Nathues, A., et al. 2012, Icarus, 220, 36

Scheirich, P., \& Pravec, P. 2009, Icarus, 200, 531

Volquardsen, E. L., Rivkin, A. S., \& Bus, S. J. 2007, Icarus, 187, 464

Walsh, K. J., \& Richardson, D. C. 2008, Icarus, 193, 553

Walsh, K. J., Richardson, D. C., \& Michel, P. 2008, Nature, 454, 188

Walsh, K. J., Delbò, M., Mueller, M., Binzel, R. P., \& DeMeo, F. E. 2012a, ApJ, 748,104

Walsh, K. J., Richardson, D. C., \& Michel, P. 2012b, Icarus, 220, 514

Walsh, K. J., Delbò, M., Bottke, W. F., Vokrouhlicky, D., \& Lauretta, D. S. 2013, Icarus, 225, 283

Wolters, S. D., Rozitis, B., Duddy, S. R., et al. 2011, MNRAS, 418, 1246 